\title{
Liter per Milligram per Meter Squared per Day
}

National Cancer Institute

\section{Source}

National Cancer Institute. Liter per Milligram per Meter Squared per Day. NCI Thesaurus.

Code C120809.

A unit of concentration equal to liter divided by milligram per meter squared per day. 